\title{
High Sensitivity Accelerometers for High Performance Seismic Attenuators
}

\author{
A. Bertolini ${ }^{1}$, R. De Salvo ${ }^{2}$, F. Fidecaro ${ }^{1}$, M. Francesconi ${ }^{1}$ \\ V. Sannibale ${ }^{2}$, A. Takamori ${ }^{3}$ \\ ${ }^{1}$ Dipartimento di Fisica dell'Università di Pisa and INFM \\ Via Buonarroti, 2 I-56127 Pisa, Italy \\ ${ }^{2}$ LIGO Project, California Institute of Technology \\ 1200 E. California Blvd., 91125 Pasadena, CA \\ ${ }^{3}$ TAMA Project, University of Tokyo \\ 7-3-1 Hongo, Bukyo-ku, Tokyo, 113-8654, Japan
}

\begin{abstract}
We present concepts and features of a new horizontal accelerometer whose mechanical design and machining process aim to improve the sensitivity in the frequency region between $10 \mathrm{mHz}$ and $1 \mathrm{~Hz}$. The expected sensitivity, less than $10^{-11} \mathrm{~m} / \mathrm{s}^{2} / \sqrt{\mathrm{Hz}}$ around $100 \mathrm{mHz}$, is a couple of orders of magnitude below the state of art limits. This accelerometer could be integrated in the active control of the LIGO II mirror seismic isolators.
\end{abstract}

We present a new highly sensitive, low frequency, horizontal accelerometer to be used in a second level of active control in an actively pre-damped (1) seismic isolator for interferometric gravity wave detectors. Our effort has been focussed on the mechanics of the device in order to reduce dissipative and thermal effects which limit the performances of the accelerometers (2) at frequencies between 0.01 and $1 \mathrm{~Hz}$. A two active stage seismic attenuator, equipped with these new accelerometers, could freeze the rms residual motion of the interferometer mirror within few nanometers, about a factor 10 better than the state of art (1).

\section{The mechanics}

The accelerometer mechanics is shaped as a small folded pendulum (3). A folded pendulum is essentially a mass suspended on one side by a simple pendulum and on the other by an inverted pendulum working antagonistically (see Figure 1a). In this way the straight pendulum positive gravitational spring constant is balanced by the inverted pendulum's negative one; by carefully shaping the mass it is also possible to lower arbitrarily the resonant frequency. In a folded pendulum the only dissipation is in the mechanics elastic flexures and in the readout/actuation system. If the elastic flexure spring constant is minimized, the mechanical losses are minimal. $Q$-factors larger than the intrinsic one of the material are achievable (4). The thermal noise acceleration spectral density is

$$
\mathrm{a}_{\mathrm{tn}}(\omega)=\frac{1}{\mathrm{Ml}} \sqrt{\frac{4 \mathrm{~K}_{\mathrm{B}} T \kappa_{\mathrm{flex}}}{\mathrm{Q} \omega}}
$$

CP523, Gravitational Waves: Third Edoardo Amaldi Conference, edited by S. Meshkov C) 2000 American Institute of Physics 1-56396-944-0/00/\$17.00 


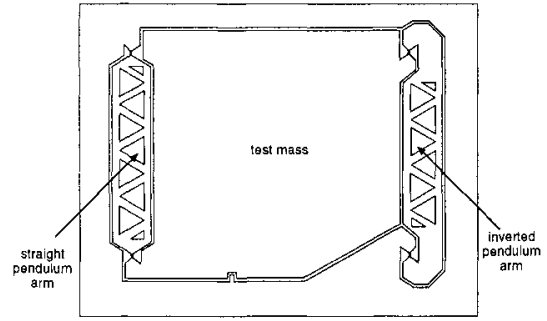

Fig. 1a. Sketch of the mechanical design of the accelerometer.

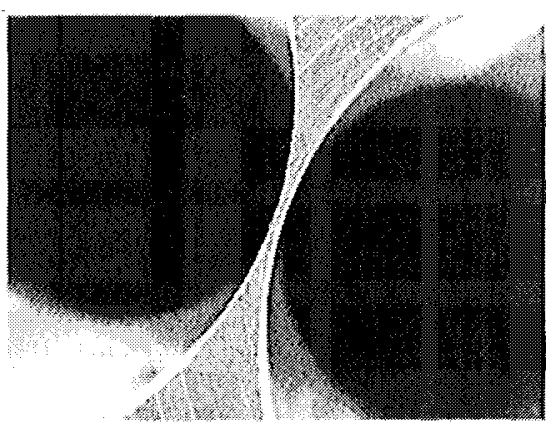

Fig. 1b. SEM image of a Cu-Be link $50 \mu \mathrm{m}$ thick machined by EDM.

where $\mathrm{M}, \mathrm{l}, \mathrm{Q}$ and $\kappa_{\mathrm{flex}}$ are respectively the mass, the pendulum arm length, the material Q-factor and the flexure angular stiffness. By using a high strength, low-losses material like $\mathrm{Cu}-\mathrm{Be}$ one can design very low $\kappa_{\text {flex }}$ flex joints $(10-20 \mu \mathrm{m}$ thick) to support masses of few kilos. In this system one can estimate a thermal noise level around $10^{-12} \mathrm{~m} / \mathrm{s}^{2} / \sqrt{\mathrm{Hz}}$ at $0.01 \mathrm{~Hz}$, with a resonant frequency of $0.2 \mathrm{~Hz}$ and a $\mathrm{Q}$ factor around 1000 . To assure high $\mathrm{Q}$ factors it is necessary to eliminate all the clamps from the mechanics eliminating the structural damping due to the so-called "stick-and-slip" mechanism. This effect is generated by boundary shear stress between contact surfaces of distinguishable mechanical parts. For this reason the accelerometer is completely machined from a single piece of $\mathrm{Cu}-\mathrm{Be}$ alloy by electric-discharge-machining (EDM). EDM allows to pre-shape the flex joints thickness to $50 \mu \mathrm{m}$ (see Figure $1 \mathrm{~b}$ ); the final thickness of $10 \mu \mathrm{m}$ will be reached by electropolishing.

\section{Control and Readout}

The instrument will be equipped with a low noise position sensor; the signal from the sensor is filtered by a PID controller and fed back to the mass through a capacitive force actuator for feedback closed-loop operation. The sensing element is a capacitive sensor working at $10 \mathrm{MHz}$, simple, rugged and with a dynamic range between $10^{6}$ and $10^{7}$; the sensor noise matches the expected thermal noise performances, $10^{-12} \mathrm{~m} / \sqrt{\mathrm{Hz}}$, with a measuring range of few microns. This results have been obtained by using the resonant phase shift readout technique (5).

\section{REFERENCES}

1. Losurdo, G., et al., Rev. Sci. Instrum. 70, 2507-2520 (1999).

2. Braccini, S., et al., Rev. Sci. Instrum. 66, 2672-2677 (1995).

3. Blair, D. G., et al., Phys. Lett. A 193, 223-226 (1994).

4. Bertolini, A., et al., LIGO Note T990777-00-D (1999).

5. Miller, G. L., et al., Rev. Sci. Instrum. 61, 1267-1272 (1990). 